\title{
INTEGRAL RESOLVENT FOR VOLTERRA EQUATIONS AND FAVARD SPACES
}

\begin{abstract}
The objective of this work is to give a characterization of the domain $D(A)$ of $A$ in terms of the integral resolvent family of the equation $x(t)=x_{0}+\int_{0}^{t} a(t-s) A x(s) d s, t \geqslant 0$, where $A$ is a linear closed densely defined operator, $a \in L_{l o c}^{1}\left(\mathbb{R}^{+}\right)$in a general Banach space $X$ and $x_{0} \in X$. Furthermore, we give a relationship between the Favard classes (temporal and frequency) for integral resolvents.
\end{abstract}

Key words: semigroups, scalar Volterra integral equations, integral resolvent families, Favard spaces

2020 Mathematical Subject Classification: 45D05, 45E05, 46B70, $47 D 06$

1. Introduction, Definitions, and Notations. Let $(X,\|\cdot\|)$ be a Banach space, $A$ a linear closed operator with dense domain $D(A)$, defined in $X$ and $a \in L_{l o c}^{1}\left(\mathbb{R}^{+}\right)$a scalar kernel. We consider the linear Volterra equation:

$$
x(t)=\int_{0}^{t} a(t-s) A x(s) d s+f(t), \quad t \geqslant 0,
$$

where $f \in \mathcal{C}\left(\mathbb{R}^{+}, X\right)$. Denote by $[D(A)]$ the domain of $A$ equipped with the graph-norm and define the convolution product $*$ of a scalar function $a$ and a vector-valued function $f$ by:

$$
(a * f)(t):=\int_{0}^{t} a(t-s) f(s) d s, \quad t \geqslant 0 .
$$

Definition 1. [11, Definition 1.1] A function $x \in \mathcal{C}\left(\mathbb{R}^{+}, X\right)$ is called:

(C) Petrozavodsk State University, 2022 
1) A strong solution of (1) if $x \in \mathcal{C}\left(\mathbb{R}^{+},[D(A)]\right)$ and (1) is satisfied.

2) Mild solution of (1) if $a * x \in \mathcal{C}\left(\mathbb{R}^{+},[D(A)]\right)$ and

$$
x=f(t)+A[a * x](t), \quad t \geqslant 0 .
$$

Obviously, every strong solution of (1) is a mild solution. Conditions under which mild solutions are strong, were studied in [11].

Definition 2. [7, Definition 2.2] Equation (1) is called well-posed if for each $v \in D(A)$ there is a unique strong solution $x(t, v)$ on $\mathbb{R}^{+}$of

$$
x(t, v)=a(t) v+(a * A x)(t), t \geqslant 0,
$$

and for any sequence $\left(v_{n}\right) \subset D(A), v_{n} \rightarrow 0$ implies $x\left(t, v_{n}\right) \rightarrow 0$ in $X$ uniformly on compact intervals.

Definition 3. [7, Definition 2.3] Let $a \in \mathcal{C}\left(\mathbb{R}^{+}\right)$be a scalar kernel. A strongly continuous family $(R(t))_{t \geqslant 0} \subset \mathcal{L}(X)$; (the space of bounded linear operators in $X)$ is called an integral resolvent for equation (1), if the following three conditions are satisfied:

(R1) $R(0)=a(0) I$.

(R2) $R(t)$ commutes with $A$, which means $R(t)(D(A)) \subset D(A)$ for all $t \geqslant 0$, and $A R(t) x=R(t) A x$ for all $x \in D(A)$ and $t \geqslant 0$.

(R3) For each $x \in D(A)$ and all $t \geqslant 0$ the resolvent equations holds:

$$
R(t) x=a(t) x+\int_{0}^{t} a(t-s) A R(s) x d s .
$$

Note that when $a(t)=1, R(t)$ corresponds to a $C_{0}$-semigroup.

If there exists an integral resolvent for (1), then a mild solution of (1) may be obtained by the formula

$$
x(t)=f(t)+A \int_{0}^{t} R(t-s) f(s) d s, \quad t \geqslant 0 .
$$

In fact, this supposes that $R(t)$ is an integral resolvent for (1); let $f \in \mathcal{C}\left(\mathbb{R}^{+}, X\right)$ and $x \in \mathcal{C}\left(\mathbb{R}^{+}, X\right)$ be a mild solution for (1). Then $R * f$ is well-defined and continuous and we obtain, from (R3) and (1):

$$
a * x=(R-A a * R) * x=R * x-R * A a * x=R * f .
$$


Hence, $R * f \in \mathcal{C}\left(\mathbb{R}^{+},[D(A)]\right)$, and from (1) we obtain

$$
x(t)=f(t)+A \int_{0}^{t} R(t-s) f(s) d s, t \geqslant 0 .
$$

The following result establishes the relation between well-posedness and existence of an integral resolvent. In what follows, $\mathcal{R}$ denotes the range of a given operator.

Theorem 1. [7, Theorem 2.4] Equation (1) is well-posed if and only if (1) admits an integral resolvent $(R(t))_{t \geqslant 0}$. If this is the case, we have, in addition, $\mathcal{R}(a * R(t)) \subset D(A)$ for all $t \geqslant 0$ and

$$
R(t) x=a(t) x+A \int_{0}^{t} a(t-s) R(s) x d s,
$$

for each $x \in X, t \geqslant 0$.

Definition 4. An integral resolvent $(R(t))_{t \geqslant 0}$ is called exponentially bounded if there exist $M>0$ and $\omega \in \mathbb{R}$, such that $\|R(t)\| \leqslant M e^{\omega t}$ for all $t \geqslant 0$ and the pair $(M, \omega)$ is called the type of $(R(t))_{t \geqslant 0}$.

The growth bound of $(R(t))_{t \geqslant 0}$ is

$$
\omega_{0}:=\inf \left\{\omega \in \mathbb{R},\|R(t)\| \leqslant M e^{\omega t}, t \geqslant 0, M>0\right\} ;
$$

if $\omega_{0}<0$, the integral resolvent is called exponentially stable.

Note that, contrary to the case of the $C_{0}$-semigroup, an integral resolvent for (1) does not need to be exponentially bounded (see [3], [11], [7]). However, there are verifiable conditions guaranteeing that (1) possesses an exponentially bounded integral resolvent.

Remark 1. Note that if $a \in \mathcal{C}^{\infty}\left(\mathbb{R}^{+}\right) \cap L^{1}\left(\mathbb{R}^{+}\right)$with $a(0)=1$ and such that $\hat{a}(\lambda)$ admits zeros with arbitrary large real part, the problem (1) cannot admit an exponentially bounded integral resolvent (For more details see [11, Page 45-46]).

We will use the Laplace transform at times; suppose that $g: \mathbb{R}^{+} \rightarrow X$ is measurable and there exist $M>0, \omega \in \mathbb{R}$, such that $\|g(t)\| \leqslant M e^{\omega t}$ for almost all $t \geqslant 0$; then the Laplace transform

$$
\widehat{g}(\lambda)=\int_{0}^{\infty} e^{-\lambda t} g(t) d t
$$


exists for all $\lambda \in \mathbb{C}$ with $\operatorname{Re} \lambda>\omega$.

A function $a \in L_{l o c}^{1}\left(\mathbb{R}^{+}\right)$is $\omega$ (resp. $\omega^{+}$)-exponentially bounded if $\int_{0}^{\infty} e^{-\omega s}|a(s)| d s<+\infty$ for some $\omega \in \mathbb{R}$ (resp. $\left.\omega>0\right)$ (see, e.g [6, p. 113]).

The following proposition, stated in [7, Theorem 3.1], establishes the relation between an integral resolvents and the Laplace transforms:

Proposition 1. Let $a \in \mathcal{C}\left(\mathbb{R}^{+}\right)$be $\omega$-exponentially bounded and let $(R(t))_{t \geqslant 0} \subset \mathcal{L}(X)$ be strongly continuous and exponentially bounded, such that the Laplace transform $\widehat{R}(\lambda)$ exists for $\lambda>\omega$. Then $(R(t))_{t \geqslant 0}$ is an integral resolvent of (1) if and only if the following conditions hold:

1) $\hat{a}(\lambda) \neq 0$ and $\frac{1}{\hat{a}(\lambda)} \in \rho(A)$, for all $\lambda>\omega$, where $\rho(A)$ is the set resolvent of $A$.

2) $K(\lambda):=\left(\frac{1}{\hat{a}(\lambda)} I-A\right)^{-1}$ called the resolvent associated to $R(t)$ satisfies:

$$
\left(\frac{1}{\hat{a}(\lambda)} I-A\right)^{-1} x=\int_{0}^{\infty} e^{-\lambda t} R(t) x d t
$$

for all $x \in X$ and all $\lambda>\omega$.

Under these assumptions, the Laplace transform of $R(\cdot)$ is well-defined and it is given by $\widehat{R}(\lambda)=K(\lambda)$ for all $\lambda>\omega$.

2. The Domain of $\boldsymbol{A}$. We have the following characterization of $D(A)$ given in [11]:

Proposition 2. Let equation (1) admit an integral resolvent family with growth bound $\omega$ (such that the Laplace transform of the resolvent exists for $\lambda>\omega)$ for $\omega$-exponentially bounded $a \in L_{l o c}^{1}\left(\mathbb{R}^{+}\right)$. Set, for $0<\theta<\frac{\pi}{2}$ and $\varepsilon>0$,

$$
\Omega_{\theta}^{\epsilon}:=\left\{\frac{1}{\widehat{a}(\lambda)}: \quad \operatorname{Re} \lambda>\omega+\varepsilon,|\arg \lambda| \leqslant \theta\right\} .
$$

Then the following characterization of $D(A)$ holds:

$$
D(A)=\left\{x \in X: \quad \lim _{|\mu| \rightarrow \infty, \mu \in \Omega_{\theta}^{0}} \mu A(\mu I-A)^{-1} x \quad \text { exists }\right\} .
$$


For more details concerning the proof of this proposition, see [11, Corollary I.1.6] and the proof of the [11, Theorem 1.4].

Without loss of generality, we may assume that $\int_{0}^{t}|a(s)| d s \neq 0$ for all $t>0$. Otherwise, we would have, for some $t_{0}>0$, that $a(t)=0$ for almost all $t \in\left[0, t_{0}\right]$ and, thus, by definition of a integral resolvent, $R(t)=0$ for $t \in\left[0, t_{0}\right]$. This implies that $A$ is bounded, which is the trivial case with $X=D(A)$.

In what follows, we will use the following assumption on $a$, such that $\int_{0}^{t}|a(s)| d s \neq 0$, for all $t>0$.

Assumption $\mathbf{H}$ : There exist $\varepsilon_{a}>0$ and $t_{a}>0$, such that for all $0<t \leqslant t_{a}$, we have:

$$
\left|\int_{0}^{t} a(t-s) a(s) d s\right| \geqslant \varepsilon_{a} \int_{0}^{t}|a(s)| d s .
$$

This is the case for functions $a$ that satisfy $a(I) \subset(0,1]$ at some interval $I=\left[0, t_{a}\right)$.

In fact, if $a(t) \in(0,1]$ for all $t \in(0,1]$, there exists $0<\varepsilon_{a} \leqslant 1$ and $t_{a}>0$, such that we have $\left|\int_{0}^{t} a(t-s) a(s) d s\right| \geqslant \varepsilon_{a} \int_{0}^{t}|a(s)| d s$ for all $0<t \leqslant t_{a}$.

For all $t \in(0,1]$, then we have $\int_{0}^{t} a(s) d s \leqslant \int_{0}^{1} a(s) d s \leqslant 1$. So,

$$
\int_{0}^{t} a(s) d s \leqslant \frac{1}{\varepsilon_{a}} \int_{0}^{t} a(t-s) a(s) d s .
$$

It is necessary and sufficient that

$$
1 \leqslant \frac{1}{\varepsilon_{a}} \int_{0}^{t} a(t-s) a(s) d s .
$$

Then we have

$$
\int_{0}^{t} a(t-s) a(s) d s \geqslant \varepsilon_{a} .
$$


Thus, $\frac{1}{\varepsilon_{a}} \int_{0}^{t} a(t-s) a(s) d s \geqslant 1 \geqslant \int_{0}^{t} a(s) d s$.

Hence, there exist $\varepsilon_{a}>0$ and $t_{a}>0$, such that we have, for all $0<t \leqslant t_{a}$ :

$$
\left|\int_{0}^{t} a(t-s) a(s) d s\right| \geqslant \varepsilon_{a} \int_{0}^{t}|a(s)| d s .
$$

For almost all reasonable functions in applications, it is easy to see that they satisfy this assumption. There are, nonetheless, examples of functions that do not.

Define the set $\widetilde{D}(A)$ as follows

$$
\widetilde{D}(A)=\left\{x \in X: \quad \lim _{t \rightarrow 0^{+}} \frac{R(t) x-a(t) x}{(a * a)(t)} \text { exists }\right\}
$$

If $|a(t)|$ is continuous and nondescreasing and

$$
\limsup _{t \rightarrow 0^{+}} \frac{\|R(t)\|}{|a(t)|}<\infty
$$

then

$$
D(A)=\left\{x \in X: \quad \lim _{t \rightarrow 0^{+}} \frac{R(t) x-a(t) x}{(a * a)(t)}=A x\right\} .
$$

This was proved in [8], [7]. Note that in the case of semigroups: $a(t)=1$ then $(a * a)(t)=t$ and in the case of cosine families: $a(t)=t$ then $(a * a)(t)=\frac{t^{2}}{2}$, we have the well-known result that $D(A)=\widetilde{D}(A)$ (see [12], [4]). In what follows, we prove that this is the case in general.

Theorem 2. Under Assumption $H$, we have $\widetilde{D}(A)=D(A)$ and

$$
\lim _{t \rightarrow 0^{+}} \frac{R(t) x-a(t) x}{(a * a)(t)}=A x .
$$

Proof. Let $x \in D(A)$ and let there exist $\varepsilon_{a}>0$ and $t_{a}>0$, such that for all $0<t \leqslant t_{a}$ we have

$$
\left|\int_{0}^{t} a(t-s) a(s) d s\right| \geqslant \varepsilon_{a} \int_{0}^{t}|a(s)| d s .
$$


For an arbitrary $\varepsilon>0$, let $t \in\left(0, t_{a}\right]$, such that $\|R(s) A x-a(s) A x\|<\varepsilon$ for all $s \in[0, t]$ due to the fact

$$
\lim _{s \rightarrow 0^{+}}\|R(s)-a(s) I\|=0
$$

by the strong continuity of $R(\cdot)$ and $a(\cdot)$ and the property $(\mathrm{R} 1)$. Then

$$
\begin{aligned}
& \left\|\frac{R(t) x-a(t) x}{(a * a)(t)}-A x\right\|= \\
& =\left\|\frac{1}{(a * a)(t)}(R(t) x-a(t) x-(a * a)(t) A x)\right\|= \\
& =\left\|\frac{1}{(a * a)(t)}\left(\int_{0}^{t} a(t-s) A R(s) x d s-\int_{0}^{t} a(t-s) a(s) A x d s\right)\right\|= \\
& =\left\|\frac{1}{(a * a)(t)}\left(\int_{0}^{t} a(t-s) R(s) A x d s-\int_{0}^{t} a(t-s) a(s) A x d s\right)\right\|= \\
& =\left\|\frac{1}{(a * a)(t)}\left(\int_{0}^{t} a(t-s)(R(s)-a(s)) A x d s\right)\right\| \leqslant \\
& \leqslant \frac{1}{|(a * a)(t)|} \int_{0}^{t}|a(t-s)|\|(R(s)-a(s)) A x\| d s \leqslant \\
& \leqslant \frac{\varepsilon}{|(a * a)(t)|} \int_{0}^{t}|a(t-s)| d s= \\
& =\frac{\varepsilon}{|(a * a)(t)|} \int_{0}^{t}|a(s)| d s \leqslant \frac{\varepsilon}{\varepsilon_{a}}<\infty
\end{aligned}
$$

due to the properties (R2), (R3) and Assumption H. This shows that $D(A) \subset \widetilde{D}(A)$.

Conversely, let $x \in \widetilde{D}(A)$ and let

$$
\lim _{t \rightarrow 0^{+}} \frac{R(t) x-a(t) x}{(a * a)(t)}=y .
$$

Under Assumption $\mathrm{H}$, we have 


$$
\begin{aligned}
& \left\|\frac{1}{(a * a)(t)} \int_{0}^{t} a(t-s) R(s) x d s-x\right\|= \\
& =\left\|\frac{1}{(a * a)(t)}\left(\int_{0}^{t} a(t-s) R(s) x d s-\int_{0}^{t} a(t-s) a(s) x d s\right)\right\| \leqslant \\
& \leqslant \frac{1}{|(a * a)(t)|} \int_{0}^{t}|a(t-s)|\|(R(s)-a(s)) x\| d s \leqslant \\
& \leqslant \frac{1}{|(a * a)(t)|} \int_{0}^{t}|a(s)| \varepsilon d s \leqslant \frac{\varepsilon}{\varepsilon_{a}} .
\end{aligned}
$$

Then $\frac{1}{(a * a)(t)} \int_{0}^{t} a(t-s) R(s) x d s \rightarrow x$ as $t \rightarrow 0^{+}$. On the other hand, by Theorem 1, we have $\int_{0}^{t} a(t-s) R(s) x d s \in D(A)$. Since $A$ is closed, we obtain, by

$$
\lim _{t \rightarrow 0^{+}} \frac{1}{(a * a)(t)} \int_{0}^{t} a(t-s) R(s) x d s=x,
$$

the following:

$\lim _{t \rightarrow 0^{+}} \frac{1}{(a * a)(t)} A \int_{0}^{t} a(t-s) R(s) x d s=\lim _{t \rightarrow 0^{+}} \frac{1}{(a * a)(t)}(R(t) x-a(t) x)=y$.

Finally, $y=A x$. Hence, $x \in D(A)$ and

$$
\lim _{t \rightarrow 0^{+}} \frac{R(t) x-a(t) x}{(a * a)(t)}=A x .
$$

From now on and in view of this result, we say that the pair $(A, a)$ is a generator of an integral resolvent $(R(t))_{t \geqslant 0}$.

3. The Favard class of $\boldsymbol{A}$. The following definition, which corresponds to a natural extension, in our context, of the Favard class, is frequently 
used in the approximation theory for semigroups and resolvent families (see e.g., [10], [4], [7], [1], [2], [9]).

Definition 5. Let equation (1) admit a bounded integral resolvent $(R(t))_{t \geqslant 0}$ on $X$, for an $\omega^{+}$-exponentially bounded $a \in L_{l o c}^{1}\left(\mathbb{R}^{+}\right)$. We define the Favard spaces (frequency and temporal) associated to $(A, a)$ as follows:

$$
\begin{aligned}
F_{a, A} & :=\left\{x \in X / \sup _{\lambda>\omega}\left\|\frac{1}{\hat{a}(\lambda)} A\left(\frac{1}{\hat{a}(\lambda)} I-A\right)^{-1} x\right\|<\infty\right\}= \\
& =\left\{x \in X / \sup _{\lambda>\omega}\left\|\frac{1}{\hat{a}(\lambda)} A K(\lambda) x\right\|<\infty\right\} .
\end{aligned}
$$

and

$$
\widetilde{F}_{a, A}:=\left\{x \in X / \sup _{0<t \leqslant 1} \frac{\|R(t) x-a(t) x\|}{|(a * a)(t)|}<\infty\right\} .
$$

Remark 2. The Favard class of $A$ with kernel a $(t)$ can be alternatively defined as the subspace of $X$ given by

$$
\left\{x \in X / \limsup _{\lambda \rightarrow \infty}\left\|\frac{1}{\hat{a}(\lambda)} A\left(\frac{1}{\widehat{a}(\lambda)} I-A\right)^{-1} x\right\|<\infty\right\} .
$$

We prove that $F_{a, A}$ is stable by $R(t)$ for any scalar kernel $a$.

Proposition 3. Let equation (1) admit a bounded integral resolvent $(R(t))_{t \geqslant 0}$ on $X$, for an $\omega^{+}$-exponentially bounded $a \in L_{l o c}^{1}\left(\mathbb{R}^{+}\right)$. We have $R(t)\left(F_{a, A}\right) \subset F_{a, A}$, for all $t \geqslant 0$.

Proof. For all $x \in D(A)$ and $t \geqslant 0$, from $(R 2)$ we have:

$$
A R(t) x=R(t) A x
$$

and, by [5, Theorem 7], $\left((\mu I-A)^{-1}\right.$ commutes with $R(t)$ for all $\left.\mu \in \rho(A)\right)$.

Since $R(t)$ is bounded, then, under Proposition 1, the following holds for all $\lambda>\omega$ :

$$
\hat{a}(\lambda) \neq 0 \text { and } \frac{1}{\hat{a}(\lambda)} \in \rho(A) .
$$

Hence, we have

$$
\left(\frac{1}{\hat{a}(\lambda)} I-A\right)^{-1} R(t)=R(t)\left(\frac{1}{\hat{a}(\lambda)} I-A\right)^{-1} .
$$


Now, if $x \in F_{a, A}$, then

$$
\sup _{\lambda>\omega}\left\|\frac{1}{\hat{a}(\lambda)} A\left(\frac{1}{\widehat{a}(\lambda)} I-A\right)^{-1} x\right\|<\infty
$$

by (6), (R2) and the boundedness of $R(t)$. We have:

$$
\begin{aligned}
\sup _{\lambda>\omega}\left\|\frac{1}{\hat{a}(\lambda)} A\left(\frac{1}{\widehat{a}(\lambda)} I-A\right)^{-1} R(t) x\right\| \leqslant \\
\\
\leqslant\|R(t)\| \times \sup _{\lambda>\omega}\left\|\frac{1}{\hat{a}(\lambda)} A\left(\frac{1}{\widehat{a}(\lambda)} I-A\right)^{-1} x\right\|<+\infty .
\end{aligned}
$$

Then $R(t) x \in F_{a, A}$ for all $t \geqslant 0$; hence, we deduce that $R(t)\left(F_{a, A}\right) \subset F_{a, A}$ for all $t \geqslant 0$.

The proof of the following proposition is immediate.

Proposition 4. The Favard classes of $A$ with kernel $a(t), F_{a, A}$, and $\widetilde{F}_{a, A}$ are Banach spaces with respect to the norms

$$
\|x\|_{F_{a, A}}:=\|x\|+\sup _{\lambda>\omega}\left\|\frac{1}{\hat{a}(\lambda)} A\left(\frac{1}{\hat{a}(\lambda)} I-A\right)^{-1} x\right\|
$$

and

$$
\|x\|_{\widetilde{F}_{a, A}}:=\|x\|+\sup _{0<t \leqslant 1} \frac{\|R(t) x-a(t) x\|}{|(a * a)(t)|}
$$

respectively.

Now we will prove that $F_{a, A}=\widetilde{F}_{a, A}$ holds for all $a \in L_{l o c}^{1}\left(\mathbb{R}^{+}\right)$satisfying Assumption $\mathrm{H}$.

Theorem 3. Let equation (1) admit a bounded integral resolvent family $(R(t))_{t \geqslant 0}$ on $X$, for an $\omega^{+}$-exponentially bounded $a \in L_{l o c}^{1}\left(\mathbb{R}^{+}\right)$, and suppose that Assumption $H$ holds. Then

$$
F_{a, A}=\widetilde{F}_{a, A}
$$

Proof. Let Assumption $\mathrm{H}$ hold: there exist $\varepsilon_{a}>0$ and $t_{a}>0$, such that for all $0<t \leqslant t_{a}$ :

$$
\left|\int_{0}^{t} a(t-s) a(s) d s\right| \geqslant \varepsilon_{a} \int_{0}^{t}|a(s)| d s .
$$


Take $x \in F_{a, A}$; let $\|R(s)\| \leqslant M$ for some $M>0$ and all $s \in[0, t]$, where $0<t \leqslant t_{a}$ and $t_{a} \geqslant 1$. We have

$$
|(a * a)(t)| \geqslant \varepsilon_{a}(1 *|a|)(t) .
$$

Then $\frac{(1 *|a|)(t)}{|(a * a)(t)|} \leqslant \frac{1}{\varepsilon_{a}}$. By Theorem 1, Remark 2, under the stability of $F_{a, A}$ by $R(t)$ :

$$
\begin{aligned}
& \frac{\|R(t) x-a(t) x\|}{|(a * a)(t)|}=\frac{1}{|(a * a)(t)|}\left\|A \int_{0}^{t} a(t-s) R(s) x d s\right\| \leqslant \\
& \leqslant \frac{1}{|(a * a)(t)|} \limsup _{\lambda \rightarrow+\infty}\left\|\int_{0}^{t} a(t-s) R(s) \frac{1}{\hat{a}(\lambda)} A\left(\frac{1}{\hat{a}(\lambda)} I-A\right)^{-1} x d s\right\| \leqslant \\
& \leqslant \frac{1}{|(a * a)(t)|}\left|\int_{0}^{t} a(s) d s\right| \limsup _{\lambda \rightarrow+\infty}\left\|\frac{1}{\hat{a}(\lambda)} A\left(\frac{1}{\hat{a}(\lambda)} I-A\right)^{-1} R(s) x\right\| \leqslant \\
& \leqslant \frac{(1 *|a|)(t)}{|(a * a)(t)|}\|R(s) x\|_{F_{a, A}} \leqslant \frac{M}{\varepsilon_{a}}\|x\|_{F_{a, A}} .
\end{aligned}
$$

Hence, we obtain $x \in \widetilde{F}_{a, A}$.

Conversely, let $x \in \widetilde{F}_{a, A}$ and set

$$
\sup _{0<t \leqslant 1} \frac{\|R(t) x-a(t) x\|}{|(a * a)(t)|}:=J_{x}<\infty .
$$

Write

$$
\frac{1}{\hat{a}(\lambda)} A\left(\frac{1}{\hat{a}(\lambda)} I-A\right)^{-1}=\frac{1}{\hat{a}(\lambda)} A K(\lambda),
$$

for all $\lambda>\omega$.

Using the integral representation of the resolvent (see Proposition 1), we obtain:

$$
\begin{aligned}
& \frac{1}{\hat{a}(\lambda)} A K(\lambda) x=\frac{1}{(\widehat{a}(\lambda))^{2}} K(\lambda) x-\frac{1}{\hat{a}(\lambda)} x= \\
&=\frac{K(\lambda) x-\hat{a}(\lambda) x}{\widehat{a * a}(\lambda)}=\int_{0}^{\infty} e^{-\lambda s} \frac{R(s) x-a(s) x}{(a * a)(s)} d s .
\end{aligned}
$$


Since $R(t)$ is bounded, by Theorem 1, and under Assumption $\mathrm{H}$, we have:

$$
\begin{aligned}
& \left\|\frac{1}{\hat{a}(\lambda)} A K(\lambda) x\right\| \leqslant\left|\int_{0}^{\infty} e^{-\lambda s} d s\right| \sup _{t>0} \frac{\|R(t) x-a(t) x\|}{|(a * a)(t)|} \leqslant \\
& \quad \leqslant \frac{1}{\lambda}\left(\sup _{t \geqslant 1} \frac{\left\|A \int_{0}^{t} a(t-s) R(s) x\right\|}{|(a * a)(t)|}+J_{x}\right) \leqslant \frac{1}{\omega}\left(\frac{L\|x\|}{\varepsilon_{a}}+J_{x}\right)<\infty
\end{aligned}
$$

with $L=\sup _{t \geqslant 1}\|A R(t)\|$. This implies $\sup _{\lambda>\omega}\left\|\frac{1}{\hat{a}(\lambda)} A K(\lambda) x\right\|<\infty$, which ends the proof.

Example 1. When $a(t)=1$, we recall that $(R(t))_{t \geqslant 0}$ corresponds to a bounded $C_{0}$-semigroup generated by $A$. In this situation, we obtain

$$
F_{1, A}=\left\{x \in X / \sup _{\lambda>0}\left\|\lambda A(\lambda I-A)^{-1} x\right\|<\infty\right\}
$$

and

$$
\widetilde{F}_{1, A}=\left\{x \in X / \sup _{t>0} \frac{\|\mathbb{T}(t) x-x\|}{t}<\infty\right\}
$$

and we have $F_{1, A}=\widetilde{F}_{1, A}$. This case is well-known (see e. g., [4]).

Example 2. Let $a(t)=b+c t$ with $b>0$ and $c>0$ with $b+c<1$, satisfying Assumption H; let equation (1) correspond to a solid in the Kelvin-Voigt model (see [11, Page 131]). Since $\hat{a}(\lambda)=\frac{b}{\lambda}+\frac{c \Gamma(2)}{\lambda^{2}}$, where $\Gamma$ denotes the Gamma function, and since $(a * a)(t)=b^{2} t+b t^{2}+\frac{c^{2}}{6} t^{3}$; then in this situation we obtain

$$
F_{a, A}=\left\{x \in X / \sup _{\lambda>0}\left\|A\left(I-\left(\frac{b}{\lambda}+\frac{c \Gamma(2)}{\lambda^{2}}\right) A\right)^{-1} x\right\|<\infty\right\}
$$

and

$$
\widetilde{F}_{a, A}:=\left\{x \in X / \sup _{0<t \leqslant 1} \frac{\|R(t) x-(b+c t) x\|}{b^{2} t+b t^{2}+\frac{c^{2}}{6} t^{3}}<\infty\right\} .
$$

And we have $F_{a, A}=\widetilde{F}_{a, A}$ thanks to Theorem 3.

Acknowledgment. The authors wish to thank the editor and the referees for thoughtful comments and constructive suggestions. 


\section{References}

[1] H. Bounit, A. Fadili. On the Favard spaces and the admissibility for Volterra systems with scalar kernel. Electronic Journal of Differential Equations, 2015, no. 42, pp. 1-21, 2015.

[2] P. L. Butzer, H. Berens. Semi-Groups of Operators and Approximation. Springer-Verlag, New York, 1967.

[3] W. Desch, J. Pruss. Counterexamples for abstract linear Volterra equations. J. Integral Equations Applications, 1993, no. 5(1), pp. 29-45.

DOI: https://doi.org/10.1216/jiea/1181075726

[4] K. J. Engel, R. Nagel. One-parameter semigroups for linear evolution equations. New York, Berlin, Heidelberg, 2000.

DOI: https://doi.org/10.1007/b97696

[5] R. Grimmer, J. Prüss. On linear Volterra equations in Banach spaces. Comp \& Maths with Appls., 1985, vol. 11, no. 1, pp. 189-205.

[6] M. Jung. Duality theory for solutions to Volterra integral equations. J.M.A.A, 1999, vol. 230, no. 1, pp. $112-134$.

DOI: https://doi.org/10.1006/jmaa.1998.6174

[7] C. Lizama, V. Poblete. On multiplicative perturbation of integral resolvent families. Journal of Mathematical Analysis and Applications, 2007, vol. 327 , no. 2 , pp.1335-1359.

DOI: https://doi.org/10.1016/j.jmaa.2006.04.087

[8] C. Lizama, J. Sanchez. On perturbation of k-regularized resolvent families. Taiwanese Journal of Mathematics, 2003, vol. 7, no. 2, pp. 217-227.

DOI: https://doi.org/10.11650/twjm/1500575059

[9] F. Maragh, H. Bounit, A. Fadili, H. Hammouri. On the admissible control operators for linear and bilinear systems and the Favard spaces. Bulletin of the Belgian Mathematical Society, 2014, vol. 21, no. 4, pp. 711-732.

DOI: https://doi.org/10.36045/bbms/1414091010

[10] J.M.A.M. van Neerven. The adjoint of a Semigroup of Linear Operators. Lecture Notes Math. 1529, Springer-Verlag, Berlin, Heidelberg, New York, 1992.

[11] J. Prüss. Evolutionary Integral Equations and Applications. BirkhäuserVerlag, Basel, 1993.

[12] Michiaki Watanabe. A new proof of the generation theorem of cosine families in Banach spaces. Houston J. Math, 1984, vol.10, no. 2, pp. 285-290. 
Received May 31, 2021.

In revised form, November 12, 2021.

Accepted November 29, 2021.

Published online December 23, 2021.

Ahmed Fadili

Laboratory LIMATI, Department of Mathematics and Informatics, Polydisciplinary Faculty, Sultan Moulay Slimane University,

Mghila, PB 592 Beni Mellal, Morocco

Email: ahmed.fadili@usms.ma

Fouad Maragh

Laboratory LAMA, Department of Mathematics, Faculty of Science, Ibn Zohr University, Boîte Postale 32/S Agadir 80000 Souss-Massa, Morocco

Email: f.maragh@uiz.ac.ma 